\title{
Prevalência e impacto da incontinência urinária na qualidade de vida em mulheres no período pós-menopausa
}

Prevalence and impact of urinary

incontinence at quality of life in

FisiSenectus . Unochapecó Ano 4, n. 2 - Jul/Dez. 2016

women in period post menopause p. $12-21$

Bianca Zezi.biank_rp@hotmail.com

Pós-Graduada em Fisioterapia na Saúde da Mulher. União das Faculdades dos Grandes Lagos Unilago, São José do Rio Preto/SP.

Hellen da Silva Camargo. hellencamargo@lie.com

Graduada em Fisioterapia. União das Faculdades dos Grandes Lagos - Unilago, São José do Rio Preto/SP.

Jaqueline Cortezia de Souza. jaqueline.cortezia@hotmail.com

Graduada em Fisioterapia. União das Faculdades dos Grandes Lagos - Unilago, São José do Rio Preto/SP.

\section{Resumo}

Introdução: A Sociedade Internacional de Continência (International Continence Society) define Incontinência Urinária (IU) como "a queixa de qualquer perda involuntária de urina" relatada pelo paciente. Objetivos: Investigar a prevalência de IU em mulheres que estão vivenciando a pós-menopausa e verificar o quanto ela interfere na qualidade de vida. Metodologia: 0 presente estudo caracterizou-se por ser uma pesquisa do tipo descritiva, no qual se verificou a prevalência da IU e a qualidade de vida em 150 participantes da cidade de Olímpia e São José do Rio Preto/SP. Os questionários utilizados foram o King's Health Questionnaire (KHQ) e o "International Consultation on Incontinence Questionnaire - Short Form" (ICIQ-SF). Resultados: 0 estudo apresentou prevalência da IU em 48,66\% na amostra estudada. Conclusão: 0 impacto da IU na qualidade de vida foi nenhum em $61,33 \%$, verificando que as avaliadas que apresentaram IU, não a consideravam como um problema que causasse impacto na sua qualidade de vida.

\section{Palavras-chave}

Incontinência Urinária; Menopausa; Fisioterapia. 


\begin{abstract}
Introduction: The International Continence Society (International Continence Society) defines Urinary Incontinence (UI) as "the complaint of any involuntary leakage of urine" reported by the patient. Objectives: To investigate the prevalence of UI in women who are experiencing postmenopausal and check how much it interferes with quality of life. Materials and methods: This study was characterized for being a descriptive research, which found the incidence of UI participants in the city of Olympia and São José do Rio Preto/ SP. The population consisted of adult women totaling 150 individuals that met the selection criteria. The questionnaires used were the King's Health Questionnaire (KHQ), and the "International Consultation on Incontinence Questionnaire - Short Form" (ICIQ-SF). Results: The study showed prevalence of UI of 48,66\% in our sample. Conclusion: The impact of urinary incontinence on quality of life was none at $61,33 \%$ showed a higher prevalence of UI to other studies of women in postmenopausal women incontinent and evaluated did not consider UI as a problem that caused impact on their quality of life.
\end{abstract}

Keywords

Urinary Incontinence; Menopause; Physical Therapy.

\section{Introdução}

$\infty \times \infty \times \infty \times \infty \times \infty \times \infty \times \infty \times \infty \times \infty \times \infty \times \infty \times \infty$

A Sociedade Internacional de Continência (International Continence Society - ICS) define Incontinência Urinária (IU) como a queixa de qualquer perda involuntária de urina relatada pelo paciente, sem necessariamente ocorrer a observação clínica do problema ${ }^{1}$.

Estima-se que 50 milhões de pessoas no mundo sofram com a IU, sendo mais comum em mulheres e podendo acometer até $50 \%$ delas em alguma fase de suas vidas. No Brasil, apesar de muitas muIheres não relatarem a presença de IU, estima-se que 11 a $23 \%$ dessa população seja incontinente, e que $20 \%$ das mulheres que vivenciam o período climatérico apresentam perda involuntária de urina, tendo manifestação $26 \%$ na fase reprodutiva elevando percentuais para $30 \%$ a $40 \%$ após a menopausa ${ }^{2,3}$.

0 aumento da expectativa de vida feminina nos últimos anos despertou maior preocupação e interesse com a saúde e a qualidade de vida das mulheres. Considerando-se que as mulheres podem viver mais de um terço de suas vidas após a menopausa, surge a necessidade de serem aprofundados conhecimentos sobre o processo natural do envelhecimento feminino e, também, sobre as patologias ou afeç̧ões que podem ocorrer facilmente nessa população ${ }^{4}$.

A IU trata-se de um problema de saúde pública encontrado em todas as faixas etárias, cujo risco aumenta com a idade, podendo determinar uma série de consequências físicas, econômicas, psicológicas, emocionais, sexuais e sociais, que irão interferir na qualidade de vida das mulheres de forma negativa, levando-as a uma mudança de comportamento. Além disso, muitas vezes desistem da prática de esportes ou de outras atividades que possam revelar seu problema, o que causa depressão, angústia e irritação ${ }^{4,5}$.

A IU pode ser classificada em três tipos mais comuns: IU por esforço, considerada como a queixa de perda involuntária de urina por esforço, a qual ocorre em situações nas quais existe um aumento da pressão intra-abdominal, como no exercício físico, tosse ou espirro; IU por hiperatividade detrusora, quando ocorre uma incapacidade de reter urina devido a um forte desejo miccional, considerada como a queixa de perda involuntária acompanhada por ou imediatamente precedido pela urgência; e IU mista, que é a queixa de perda involuntária associada com urgência e, também, com a execução de esforço, tosse ou espirro ${ }^{6,7}$.

Os fatores de risco para o desenvolvimento da IU incluem: idade avançada, raça branca, obesidade, partos vaginais, deficiência estrogênica, condições associadas a aumento da pressão abdominal, tabagismo, neuropatias, cirurgias ginecológicas, menopausa, suporte pélvico enfraquecido, hipermobilidade anatômica, frouxidão tecidual e prolapso vesical ou uterino ${ }^{4,6,8}$.

Dentre as causas da IU, a menopausa se apresenta como um dos fatores de risco para o seu 
desenvolvimento. O climatério é uma etapa marcante do envelhecimento feminino caracterizada pelo estabelecimento de estado fisiológico de hipoestrogenismo progressivo e culminando com a interrupção definitiva dos ciclos menstruais. Inicia-se normalmente entre 35 e 40 anos, estendendo-se até os 65 anos, sendo frequentemente acompanhadas por sintomas característicos como sudorese, fogachos, alterações de humor e sono. Além de fadiga, perda de elasticidade da pele e resistência dos ossos, e dificuldades na esfera emocional e social, consequentemente interferindo na qualidade de vida dessas mulheres ${ }^{9,10}$.

Marcada por mudanças na função ovariana, a menopausa representa redução dos níveis de estrogênio circulante e é considerada um evento único que marca a transição do período reprodutivo para o não reprodutivo. A menopausa natural é definida pela Organização Mundial de Saúde (OMS) como sendo 12 meses consecutivos de amenorreia, sem outra causa patológica ou psicológica9,10.

0 estrogênio aumenta o trofismo e a vascularização dos músculos do assoalho pélvico (MAP) e os receptores desse hormônio são encontrados na vagina, bexiga, uretra e no MAP. Receptores de estrogênio exercem um papel importante no mecanismo de suporte da pelve, controlando a síntese e a degradação de colágeno. Além disso, os tecidos do mecanismo de continência urinária feminina são sensíveis ao estrogênio. 0 estrogênio pode afetar a continência, melhorando a resistência da uretra através do aumento do número de vasos periuretral que representam um terço da pressão uretral, e pode reduzir a frequência, amplitude das contrações do detrusor e, assim, aumentar o limiar sensorial da bexiga e promover o relaxamento do músculo detrusor. Devido à redução na produção desse hormônio, o MAP se torna mais delgado, seco e menos elástico, tornando-se mais suscetível a irritações e é provável que sua deficiência possa levar à IU. Isso é respaldado pela alta prevalência de IU na menopausa9,11.

Outro fator importante na continência é o músculo elevador do ânus que funciona como um eventual mecanismo de fechamento em momentos de aumento da pressão intra-abdominal, ajudando a manter a pressão uretral máxima maior que a pressão vesical. No entanto, com o avançar da idade, há um comprometimento do suporte neuromuscular do assoalho pélvico, causando progressiva atrofia dos tecidos de sustentação, o que prejudica a disposição das fibras do músculo elevador do ânus ${ }^{12}$.

A ICS recomenda que a fisioterapia seja a primeira opção de tratamento em caso de IU, por ser um método não invasivo, de baixo custo e sem efeitos colaterais. Além disso, 50\% das cirurgias de IU poderiam ter sido evitadas se fossem realizados exercícios do assoalho pélvico. Após definido o tipo de IU, o tratamento se baseia em intervenções no estilo de vida e o treinamento do MAP por meio dos recursos oferecidos pela fisioterapia ${ }^{13}$.

Dentre os recursos utilizados estão os cones vaginais, treinamento comportamental e cinesioterapia que representam uma forma prática e simples para fortalecimento do MAP; eletroestimulação endovaginal por meio de eletroestimuladores pequenos e práticos, intravaginais ou transanais, utilizados com o objetivo de inibir o músculo detrusor diminuindo o número de miç̧ões e aumentar a força muscular; biofeedback permite que o paciente realize exercícios isolando a musculatura do assoalho pélvico, o que proporciona um aumento na força mensurada; estrogénios tópicos vaginais apresenta uma eficácia muito baixa na melhoria da IU, no entanto, dado o seu baixo custo e os outros benefícios que proporcionam para a mulher em idade pós-menopausa, são uma opção como terapêutica adjuvante neste grupo etário $8,14,15,16,17$.

Os objetivos desta pesquisa são investigar a prevalência de IU em mulheres que estão vivenciando a pós-menopausa e verificar o quanto ela interfere na qualidade de vida.

\section{Materiais e métodos}

$\infty<\infty<\infty<\infty<\infty<\infty<\infty<\infty<\infty<\infty<\infty<\infty<\infty<\infty<\infty<\infty$

0 presente estudo caracterizou-se por ser uma pesquisa do tipo descritiva, no qual se pretendia verificar a incidência da IU em participantes das cidades de São José do Rio Preto e Olímpia, ambas localizadas no Estado de São Paulo. A coleta de dados foi realizada em dois meses, precedida pela aprovação do projeto de pesquisa pelo Comitê de Ética da UNILAGO Ofício n ${ }^{\circ}$ 140/15. 
A pesquisa foi realizada em seis meses. Para a amostra, foram avaliadas 150 voluntárias do gênero feminino com idade entre 50 e 85 anos (média de 63 anos) e que se enquadravam nos critérios de seleção: ter idade igual ou superior a 50 anos, encontrar-se no período pós-menopausa em que o último período menstrual ocorreu há pelo menos 12 meses antes de participar do estudo, ter condições físicas e mentais para responder ao questionário e consentir em participar do estudo.

Inicialmente, as participantes recebiam as instruções explicativas da pesquisa, seguidas de instruções em relação aos questionários, e eram convidadas a participar da pesquisa.

Foram utilizados os questionários de Impacto de Incontinência Urinária International Consultation on Incontinence Questionnaire - Short Form - ICIQ-SF e o King's Health Questionnaire - KHQ validado por Kelleher et al. ${ }^{18}$. Os questionários foram entregues às voluntárias juntamente com uma caneta para seu preenchimento e o termo de consentimento livre e esclarecido.

O ICIQ-SF, instrumento breve, traduzido para o português, avalia o impacto da IU na qualidade de vida e a qualificação da perda urinária dos pacientes analisados. É composto por quatro questões relacionadas à frequência, à gravidade da perda urinária e a seu impacto na qualidade de vida. O ICIQ Escore (ICIQ E) é a soma dos escores das questões três, quatro e cinco e varia de 0 a 21 . É considerado diagnóstico de IU qualquer pontuação superior a zero. 0 impacto na qualidade de vida foi definido de acordo com o escore da questão 5: (0) nada; (1-3) leve; (4-6) moderado; (7-9) grave; (10) muito grave ${ }^{19}$.

O KHQ é um instrumento que avalia o impacto dos sintomas do trato urinário baixo na qualidade de vida de mulheres. É composto de 21 questões, divididas em oito domínios: percepção geral de saúde (um item), impacto da IU (um item), limitações de atividades diárias (dois itens), limitações físicas (dois itens), limitações sociais (dois itens), relacionamento pessoal (três itens), emoções (três itens), sono/disposição (dois itens). Além desses domínios, existem duas outras escalas independentes: uma avalia a gravidade da IU (medidas de gravidade) e outra a presença e a intensidade dos sintomas urinários (escala de sintomas uriná(rios) $)^{18}$.

Essas escalas são graduadas em quatro opções de respostas (nem um pouco, um pouco, moderadamente, muito ou nunca, às vezes, frequentemente, o tempo todo), exceção feita ao domínio percepção geral de saúde com cinco opções de respostas (muito boa, boa, regular, ruim, muito ruim) e ao domínio relações pessoais (não aplicável, nem um pouco, um pouco, moderadamente e muito $)^{18,20}$.

O KHQ é pontuado por cada um de seus domínios, não havendo, portanto, escore geral. A todas as respostas são atribuídos valores numéricos, somados e avaliados por domínios. As respostas foram baseadas em uma escala numérica crescente e proporcional à intensidade da queixa $(0=$ não/ não se aplica; $1=$ um pouco/às vezes; $2=$ mais ou menos/várias vezes; $3=$ muito/sempre), exceção feita ao domínio percepção geral de saúde, que tem cinco opções de resposta: muito boa, boa, regular, ruim, muito ruim. Os escores variam de 0 a 100 e quanto maior a pontuação obtida, pior é a qualidade de vida relacionada àquele domínio ${ }^{18,20}$.

Para análise dos dados, utilizou-se o Microsoft Office Excel 2007.

\section{Resultados}

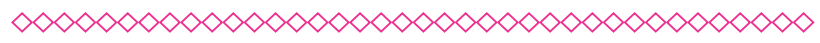

Durante o estudo, foram incluídas 150 mulheres com idade média de 63 anos, sendo grande maioria da raça branca $(84 \%)$ e casada $(60,7 \%)$. As características das voluntárias foram descritas na Tabela 1.

O Gráfico 1 apresenta a prevalência geral de IU na amostra populacional, de acordo com o ICIQ-SF foi de $48,66 \%$ (73 de 150).

De acordo com o Gráfico 2, na questão em que se avaliou a situação em que ocorre a perda de urina, as participantes poderiam assinalar mais de uma questão. Os resultados obtidos foram: $12,66 \%$ antes de conseguir chegar ao banheiro; $32 \%$ quando tosse ou espirra; $6 \%$ quando estão dormindo; $8 \%$ durante atividade física; $2 \%$ quando de urinar e estar vestida; $6,66 \%$ sem razão aparente; e 2,66\% constantemente. 
0 impacto na qualidade de vida foi avaliado pelas respostas à questão cinco, sendo (0) nada 61,33\%; (1-3) leve 16\%; (4-6) moderado 13,34\%; (7-9) grave $4,66 \%$; (10) muito grave $4,67 \%$, como demonstrado no Gráfico 3.

Em relação à escala de sintomas urinários do $\mathrm{KHQ}$, foi investigado isoladamente o quanto alguns sintomas afetavam as mulheres. Na Tabela 2, destaca-se o escore mais elevado no domínio impacto da IU (escore médio de 2.30) e o mais baixo no domínio relacionamento pessoal (escore médio de 0.6). 0 domínio limitações de atividades diárias apresentou escore médio de 1.92, limitações físicas e sociais 1.5 , presença e a intensidade dos sintomas urinários 2.26, emoções 1.92, sono/energia 1.24 e gravidade da IU 2.08 .

\section{Discussão}

$\infty<\infty<\infty<\infty<\infty<\infty<\infty<\infty<\infty<\infty<\infty<\infty<\infty<\infty<\infty<\infty$

A ocorrência de IU na amostra deste estudo foi superior $(48,66 \%)$ a de outros estudos realizados com mulheres no período pós-menopausa, considera-se uma prevalência alta já que quase $50 \%$ das mulheres avaliadas apresentavam queixa de IU. Constatou-se que $23,33 \%$ destas perdiam apenas uma vez por semana e que $35,33 \%$ delas relataram perder uma pequena quantidade. Ao realizar o questionamento sobre a frequência da IU, pôde-se perceber o constrangimento das participantes ao responderem; assim, acredita-se que o número de pessoas incontinentes seja maior do que o que foi relatado.

Vários estudos com mulheres no período pós-menopausa têm explorado a relação dessa fase com a IU, como o de Pedro et al. ${ }^{21}$, publicado em 2003, na cidade de Campinas/SP, Brasil, abrangendo 456 mulheres no período de climatério, que evidenciou uma prevalência de $27,4 \%$ de IU e o de Guarisi et al. ${ }^{22}$ que analisaram 456 mulheres que apresentava idade entre 45 a 60 anos, 35\% se queixaram de perda de urina por esforços, e 10,7\% referiram que esse sintoma ocorria sempre.

Da mesma maneira que a presente pesquisa observou a prevalência de IU entre as mulheres que estão no período pós-menopausa, Tamanini et al. ${ }^{19}$ realizaram um estudo epidemiológico em São
Paulo/SP, Brasil, com 2.143 idosos com mais de 60 anos de idade, e encontraram uma prevalência da IU de $26,2 \%$ entre as mulheres, concluindo que essa condição está relacionada com o avançar da idade e com as limitações funcionais consequentes.

Resultados semelhantes ao deste estudo foram encontrados no estudo de Sebben e Filho ${ }^{23}$, que teve por objetivo investigar a incidência da IU em participantes do município de Passo Fundo/ RS, Brasil, com a aplicação do ICIQ-SF respondido pelos participantes da amostra. Os resultados obtidos foram de $55,2 \%$ dos 250 participantes apresentando IU. A maioria dos participantes do estudo considerava a IU como um problema que não afetava muito a sua qualidade de vida, ao mesmo tempo em que desconhecia informações em relação ao tratamento.

Em um estudo epidemiológico realizado no Chile em mulheres com idade superior a 30 anos, Ardila ${ }^{24}$, utilizou como avaliação um questionário autoaplicado, e o índice de Sandvik para calcular a severidade da IU. Neste estudo, a maior prevalência de IU foi encontrada em mulheres entre 46 e 59 anos, mas a prevalência em mulheres de 60 anos ou mais foi um pouco menor. Isso pode estar relacionado a vários fatores, aspecto cultural, vergonha ou a ideia de que a IU é um aspecto "normal" ou inevitável do envelhecimento.

As prevalências de IU em mulheres na pré e pós-menopausa têm sido muito estudadas e os resultados têm confirmado associação significativa, com índices que variam de $46 \%$ a $64 \%$. 0 hipoestrogenismo na pós-menopausa predispõe a muIher à IU e contribui para sintomas urinários como aumento da frequência, urgência e disúria ${ }^{25}$.

Omli et al. ${ }^{26}$ analisaram a IU e o declínio funcional de 770 indivíduos, durante o período de 11 anos, sendo eles incontinentes e continentes. 0 estudo foi dividido em dois períodos, a prevalência de IU foi de $24 \%$ (183) no início do estudo e, após 11 anos de acompanhamento, 353 (46\%) das mulheres relataram que tinham IU. 0 grande aumento da IU pode ser explicado tanto por um aumento na deficiência física e no aumento da prevalência de doenças como diabetes e acidente vascular cerebral, que são conhecidos como fatores de risco para a IU em idosos. Em média 11 anos de acompanhamento mostraram uma associação 
significativa entre a IU e declínio nas atividades da vida diária.

No presente estudo, observou-se que $32 \%$ das avaliadas relataram perda de urina durante a tosse ou espirro, portanto considera-se que o tipo de IU prevalecente entre as avaliadas foi a por esforço.

Lopes e Higa ${ }^{27}$ realizaram um estudo descritivo, onde foram analisados prontuários de 164 mulheres que apresentavam queixa de IU e participaram do Programa de Reabilitação do Assoalho Pélvico na cidade de Campinas/SP, Brasil. Os instrumentos utilizados foram os questionários de ICQ-SF, KHQ e SF-36. Como resultados obtiveram alterações nas atividades sexuais $(40,9 \%)$, sociais $(33,5 \%)$, domésticas $(18,9 \%)$ e ocupacionais $(15,2 \%)$, sendo a IU mista e a de urgência as que mais afetavam a vida das mulheres.

Com o objetivo de verificar a prevalência da IU entre mulheres idosas segundo a prática de atividade física (AF) regular, Virtuoso et al. ${ }^{28}$ avaliaram 209 idosas, divididas em três grupos, segundo o nível de AF. A presença de IU na amostra total foi de $33,3 \%$, sendo a menor prevalência entre as idosas mais ativas (28,9\%). A prática de ginástica associou-se com a ausência de IU. Em 43,5\% da amostra, o início dos sintomas de gravidade deu-se após a menopausa.

A qualidade de vida das mulheres incontinentes pode ser afetada de diversas maneiras. Em um aspecto geral, referem limitações em níveis físicos (praticar esporte, carregar objetos), alterações do sono como a noctúria e a enurese noturna, podendo levar a cansaço, alterações nas atividades sociais, ocupacionais e domésticas, influenciando negativamente o estado emocional e a vida sexual. Além disso, a IU pode provocar desconforto social e higiênico, pelo medo da perda urinária, pelo cheiro de urina, pela necessidade de utilizar protetores (absorventes) e de trocas mais frequentes de roupas $^{20,29}$.

Verificou-se, neste estudo, por meio do ICIQ-SF, que as mulheres que apresentam IU não notaram relevância no impacto na qualidade de vida (61,33\%). E, por meio do KHQ, observou-se o mesmo resultado no impacto da IU (média de 2,30), assim como não foram constatados resultados significantes nos domínios limitações de atividades diárias, limitações físicas e sociais, presença e a intensidade dos sintomas urinários, emoções, sono/energia, relacionamento pessoal e gravidade da IU. Evidenciando, portanto, uma contradição, embora a IU seja um dos agravos mais frequentes à saúde da mulher no período pós-menopausa e afete sua qualidade de vida.

Semelhantemente, Lazari et al. $^{3}$ realizaram um estudo descritivo que pretendia investigar a qualidade de vida de 22 idosas com IU cuja média de idade foi de 72,9 anos, utilizando o questionário ICIQ-SF e uma escala tipo Likert utilizada para quantificar a interferência da perda de urina na qualidade de vida, e constataram que para $27,5 \%$ dessas idosas nada significava e para $22,7 \%$ delas representava de grande interferência.

Abreu et al..$^{30}$ realizaram um estudo qualitativo com 12 idosas de 60 anos ou mais, que foram submetidas ao tratamento de IU, com intuito de verificar a qualidade de vida delas, sendo realizado por meio de entrevistas individuais semiestruturadas. A análise do conteúdo das entrevistas permitiu concluir que, apesar de a IU estar presente na vida das idosas, trazendo prejuízos psicológicos e sociais, a qualidade de vida foi positivamente percebida.

Rett et al. ${ }^{20}$ realizaram um estudo que comparou a qualidade de vida antes e após tratamento fisioterápico de 26 mulheres com IU de esforço, sendo feita a análise por meio do questionário $\mathrm{KHQ}$. Ao final do tratamento, observou-se que, em relação à alta frequência urinária, menos de metade da amostra, 12 (46, 2\%) mulheres, não apresentava mais o sintoma.

Constatou-se, no presente estudo, uma prevalência da IU de 48,66\% nas mulheres avaliadas; porém, não foram observadas alterações na qualidade de vida dessas participantes.

\section{Conclusão}

$\infty \times \infty \times \infty \times \infty \times \infty \times \infty \times \infty \times \infty \times \infty \times \infty \times \infty \times \infty \times \infty$

Verificou-se que a prevalência da IU na população estudada foi superior à encontrada em outros estudos realizados com mulheres no período pós-menopausa, além de se constatar que as avaliadas que apresentaram IU não a consideravam como um problema que causasse impacto na sua qualidade de vida. 
O baixo impacto na qualidade de vida das participantes pode ter ocorrido devido à média de idade das participantes não ter sido muito alta. Muitas mulheres estão há pouco tempo na menopausa, e a redução dos níveis de estrógeno pode não ter sido tão significativa ao ponto de a IU ter afetado sua qualidade de vida. Porém, vale lembrar que a IU piora de forma progressiva quando não tratada e, em longo prazo, estas mesmas muIheres podem apresentar piora do quadro e sofrerem grande impacto na qualidade de vida. Pôde-se perceber o constrangimento das participantes ao responderem ao questionário; assim, acredita-se que o número de pessoas incontinentes seja maior do que o que foi relatado pelo presente estudo, demonstrando que a IU está relacionada com a menopausa.

\section{Referências}

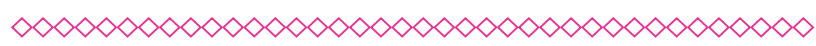

1. Haylen BT, Ridder D, Freeman RM, Swift EE, Berghmans B, Lee J, Monga A, Petri E, Rizk $D E$, Sand $P K$, Schaer GN. An International Urogynecological Association (IUGA) / International Continence Society (ICS) joint report on the terminology for female pel-vic floor dysfunction. Neurourology and Urodynamics. 2010; 29(1):4-20.

2. Abrams $P$, Cardozo L, Fall M, Griffiths $D$, Rosier $\mathrm{P}$, Ulmsten $U$, Van Kerrebroeck $\mathrm{P}$; Victor A, Wei A. The standardi-zation of terminology in lower urinary tract function: report from the standardization sub-committee of the International Continence Society. Urology. 2003; 61(1):37-49.

3. Lazari ICF, Lojudice DC, Marota AG. Avaliação da qualidade de vida de idosas com incontinência urinária: idosas institucionalizadas em uma instituição de longa permanência. Rev. Bras. Geriatr. Gerontol. 2009; 12(1):103-112.

4. Berlezi EM, Dal Bem A, Antonello C, Leite MT, Bertolo EM. Incontinência urinária em mulheres no período pós-menopausa: um problema de saúde pública. Revista Brasileira de Geriatria e Gerontologia. 2009; 12(2):159-173.
5. Berquó MS, Ribeiro MO, Amaral RG. Fisioterapia no tratamento da incontinência urinária feminina. Feminina. 2009; 37(7):385-388.

6. Mourão FAG, Lopes LN, Vasconcellos NPC, Almeida MBA. Prevalência de queixas urinárias e o impacto destas na qualidade de vida de mulheres integrantes de grupos de atividade física. Acta Fisiatrica. 2008; 15(3):170-175.

7. Gomes GVS, Genivaldo D. Incontinência urinária de esforço em mulheres pertencentes ao Programa de Saúde da Família de Dourados (MS). Rev. Assoc. Med. Bras. 2010; 56(6):649-654.

8. Kubagawa LM, Pellegrini JRF, Lima VP, Moreno AL. A eficácia do tratamento fisioterapêutico da incontinência urinária masculina após prostatectomia. Revista Brasileira de Cancerologia. 2006; 52(2):179-183.

9. Batista RLA, Souza FO, Dias LAR, Silva ACJSR, Freitas MMS, De As MFS, Ferreira CHJ. Revisão sistemática das influências do hipoestrogenismo e do treinamento sobre a incontinência urinária. Feminina. 2010; 38(3):135-140.

10. LorenziI DRS, Baracat EC, Saciloto B, Padilha Jr. I. Fatores Associados á qualidade de vida após menopausa. Revista Associação Medica Brasileira. 2006; 52(5):312-317.

11. Weber MA, Kleijn MHM, Langendam M, Limpens J, Heineman MJ, Roovers JP. Local Oestrogen for Pelvic Floor Disorders: A Systematic Review. PLoS One. 2015 Sep 18;10(9).

12. Souza CEC, Lima RMB, Lidia MA, Pereira RW, Moura TK, Oliveira RJ. Estudo comparativo da função do assoalho pélvico em mulheres continentes e incontinentes na pós-menopausa. Rev. bras. Fisioter. 2009; 13(6):535-541.

13. Glisol SFN, Girelli P. Importância da fisioterapia na conscientização e aprendizagem da contração da musculatura do assoalho pélvico em mulheres com incontinência urinária. Rev Bras Clin Med. São Paulo. 2011 Nov/Dez; 9(6):408-413.

14. Bernardes NO, Peres FR, Souza ELB; Souza OL. Métodos de Tratamento Utilizados na Incontinência Urinária de Esforço Genuína: um Estudo Comparativo entre Cinesioterapia e 
Eletroestimulação Endovaginal. Revista brasileira de ginecologia e obstetrícia. 2000; 22(1):49-54.

15. Botelho F, Silva C, Cruz F. Incontinência Urinária Feminina. Acta Urológica. 2007; 24(1):79-82.

16. Castro AP, Pereira VS, Serrão PRMS, Driusso P. Eficácia do biofeedback para o tratamento da incontinência urinária de esforço: uma revisão sistemática. Scientia Medica. 2010; 20(3):257263.

17. Oliveira $K A C$, Rodriguez $A B C$, Paula $A B$. Técnicas fisioterapêuticas no tratamento e prevenção da incontinência urinária de esforço na mulher. Revista Eletrônica F@pciência. 2007; 1(1):31-40.

18. Kelleher CJ, Cardozo LD, Khullar V, Salvatore S. A new questionnaire to assess the quality of life of urinary incontinent women. Br J Obstet Gynaecol. 1997; 104:1374-1379.

19. Tamanini JTN, Lebrão ML, Duarte YAO, Santos $J L F$, Laurenti R. Analysis of the prevalence of and factors associated with urinary incontinence among elderly people in the Municipality of São Paulo, Brazil: SABE Study (Health, Wellbeing and Aging). Cadernos de Saúde Pública. 2009; 25(8):1756-1762.

20. Rett MT, Simões JA, Herrmann V, Gurgel MSC. Qualidade de vida em mulheres após tratamento da incontinência urinária de esforço com fisioterapia. Rev Bras Ginecol Obstet. 2007; 29(3):134-140.

21. Pedro AO, Pinto Neto AM, Costa Paiva LHS, Osis MJD, Hardy EE. Síndrome do climatério: inquérito populacional domiciliar em Campinas. Rev Saúde Pública. 2003; 37(6):735-742.

22. Guarisi TN, Aarão MP, Osis MJ, Pedro AO, Paiva LHC, Faundes A. Procura de Serviço Médico por Mulheres com Incontinência Urinária. RBGO. 2001; 23(7):439-443.
23. Sebben V, Tourinho Filho H. Incidência da incontinência urinária em participantes do Creati do município de Passo Fundo/RS. RBCEH. 2008; 5(2):101-109.

24. Ardila OR. Caracterización clínica de la incontinencia urinaria y factores asociados en usuarias de la Unidad de la Mujer del Centro de Salud Familiar "Ultraestación" en la ciudad de Chillán, Chile. Rev. Méd. Chile. 2015;143(2):203212.

25. Higa R, Lopes MHBM, Reis MJ. Fatores de risco para incontinência urinária na mulher. Rev. esc. enferm. USP. 2008; 42(1):187-192.

26. Omli R, Hunskaar S, Mykletun A, Romild $U$, Kuhry $E$. Urinary incontinence and risk of functional decline in older women: data from the Norwegian HUNT-study. BMC Geriatrics. 2013 May; 13(1):47.

27. Lopes MHBM, Higa R. Restrições causadas pela incontinência Restrições causadas pela incontinência urinária à vida da mulher urinária à vida da mulher. Rev Esc Enferm USP. 2006; 40(1):34-41.

28. Virtoso JF, Mazo GZ, Menezes EC. Prevalência, tipologia e sintomas de gravidade da incontinência urinária em mulheres idosas segundo a prática de atividade física. Fisioter. Mov., Curitiba. 2012; 25(3):571-582.

29. Auge, AP; Zucchi CM, Costa FMP, Nunes $K$, Cunha LPM, Silva PVF, Ramos T. Comparações entre os índices de qualidade de vida em mulheres com incontinência urinária submetidas ou não ao tratamento cirúrgico. Rev Bras Ginecol Obstet. 2006; 28(6):352-357.

30. Abreu N; Baracho OES, Tirado MGA, Dias RC. Qualidade de vida na perspectiva de idosas com incontinência urinária. Rev. bras. fisioter. 2007; 11(6):429-436. 


\section{Anexos}

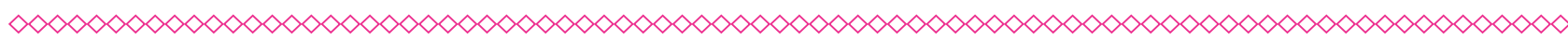

Tabela 1 - Características das participantes.

\begin{tabular}{lc} 
& \\
Gênero $(q)$ & Caracteristicas das Participantes \\
Idade (anos) & 150 \\
Raça (branca/negra/ parda) & 63 \\
Estado civil (solteira/casada/divorciada/viúva) & $84 \% / 12 \% / 4 \%$ \\
\hline
\end{tabular}

(clique para voltar ao texto)

Gráfico 1 - Prevalência geral da IU.

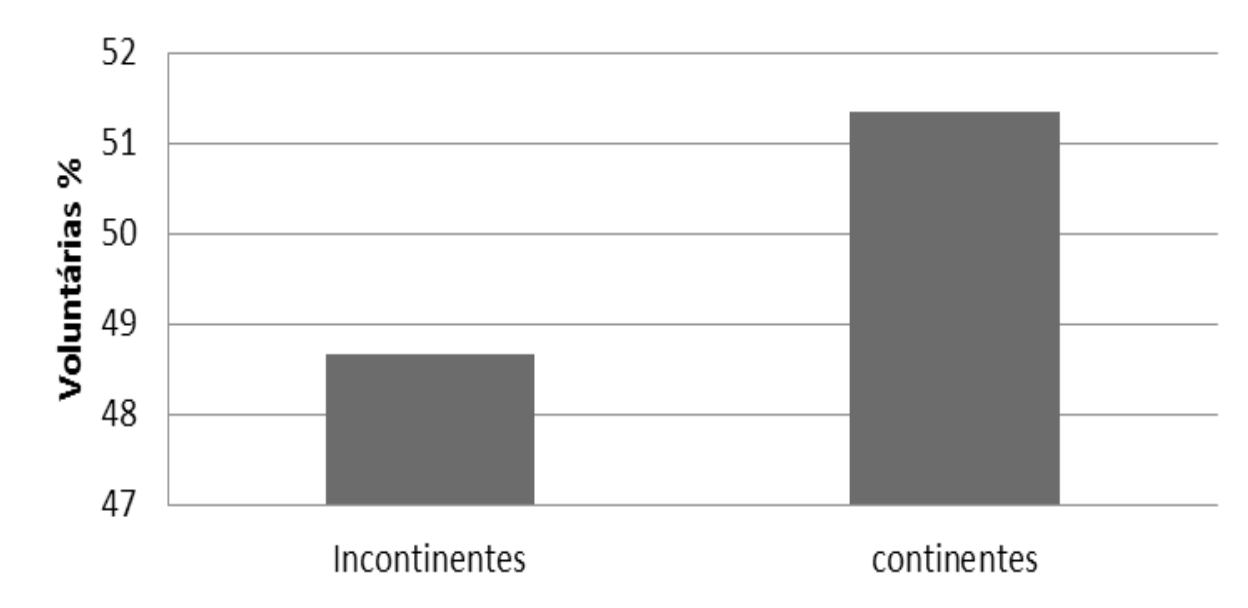

(clique para voltar ao texto)

Gráfico 2 - Situação em que ocorre a perda de urina.

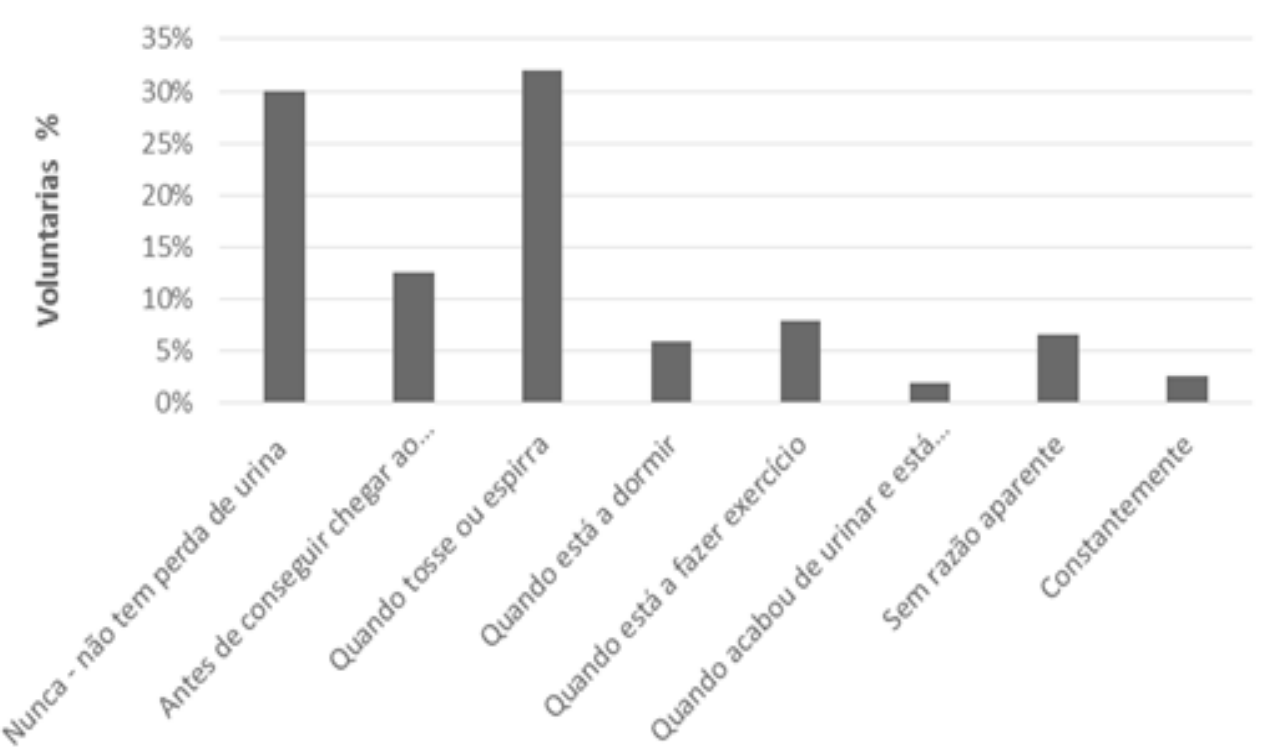

(clique para voltar ao texto) 
Gráfico 3 - Impacto na qualidade de vida.

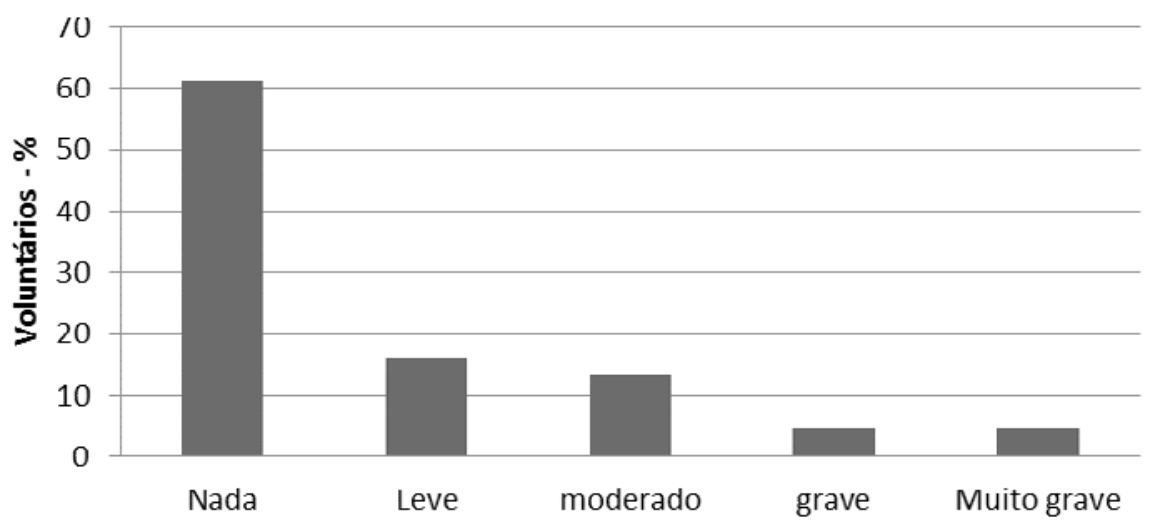

(clique para voltar ao texto)

Tabela 2 - Domínios do KHQ.

\begin{tabular}{lc} 
Domínios do KHQ & Média \\
Impacto da incontinência & 2.30 \\
Limitações de atividades diárias & 1.92 \\
Limitações físicas e sociais & 1.5 \\
Relacionamento pessoal & 0.6 \\
Presença e a intensidade dos sintomas urinários & 2.26 \\
Emoções & 1.92 \\
Sono/energia & 1.24 \\
Gravidade da IU & 2.08 \\
\hline
\end{tabular}

